EPJ Web of Conferences 37, 05004 (2012)

DOI: $10.1051 /$ epjconf/20123705004

(C) Owned by the authors, published by EDP Sciences, 2012

\title{
Search for the $K_{S} \rightarrow 3 \pi^{0}$ decay with the KLOE detector
}

\author{
M. Silarski ${ }^{\mathrm{a}}$ on behalf of the KLOE -2 Collaboration ${ }^{\mathrm{b}}$ \\ Institute of Physics, Jagiellonian University, PL-30-059 Cracow, Poland
}

\begin{abstract}
The $K_{S} \rightarrow 3 \pi^{0}$ decay is a pure $C \mathcal{P}$ violating process which, assuming CPT invariance, gives access to the $C^{\mathscr{P}}$ - violating phase of the weak decay amplitude into I=1 final states. This decay has not been observed so far, and the best upper limit on the branching ratio $B R\left(K_{S} \rightarrow 3 \pi^{0}\right)<1.2 \cdot 10^{-7}$ is two orders of magnitude larger than predictions based on the Standard Model. In this article we present the search for the $K_{S} \rightarrow 3 \pi^{0}$ decay performed with the KLOE detector operating at the DAФNE $\phi$-factory at the Frascati Laboratory. We describe the analysis techniques used for the background rejection and signal events selection leading to the result of an almost five times lower upper limit on the $K_{S} \rightarrow 3 \pi^{0}$ branching ratio. We also discuss the perspectives for a new measurement using the KLOE - 2 apparatus equipped with a new inner tracker and the calorimeters at low $\theta$ angle.
\end{abstract}

\section{Introduction}

The $C \mathcal{P}$ symmetry violation was discovered in 1964 by Christenson, Cronin, Fitch and Turlay while studying the regeneration of the neutral $K$ mesons [1]. Since this unexpected discovery the $C \mathcal{P}$ violation parameters of neutral kaons have been measured with a good precision by several experiments, and at present the main experimental effort is focused on studies of the neutral $B$ and $D$ meson systems. However, there are still several interesting open issues in the kaon physics. One of them is the $K_{S} \rightarrow 3 \pi^{0}$ decay which, assuming the $C \mathcal{P T}$ invariance, allows one to investigate the $C \mathcal{P}$ - violating phase of the weak decay amplitude into $I=1$ final states. Despite several direct searches $[2$, $3]$ and $K_{S} K_{L}$ interference studies [4,5], this decay remains undiscovered and the best upper limit on the branching ratio $B R\left(K_{S} \rightarrow 3 \pi^{0}\right)<1.2 \cdot 10^{-7}[3,7]$ is still two orders of magnitude larger than the predictions based on the Standard Model: $B R\left(K_{S} \rightarrow 3 \pi^{0}\right) \sim 2 \cdot 10^{-9}$ [3].

In this article we briefly describe the search of the $K_{S} \rightarrow 3 \pi^{0}$ decay based on the data sample gathered in 2004 - 2005 with the KLOE detector operating at the $\phi$ - factory DAФNE of the Frascati Laboratory.

a e-mail: Michal.Silarski@lnf.infn.it

b The KLOE - 2 Collaboration: D. Babusci, D. Badoni, I. Balwierz, G. Bencivenni, C. Bini, C. Bloise, V. Bocci, F. Bossi, P. Branchini, A. Budano, L. Caldeira Balkeståhl, G. Capon, F. Ceradini, P. Ciambrone, E. Czerwiński, E. Dané, E. De Lucia, G. De Robertis, A. De Santis, A. Di Domenico, C. Di Donato, R. Di Salvo, D. Domenici, O. Erriquez, G. Fanizzi, A. Fantini, G. Felici, S. Fiore, P. Franzini, P. Gauzzi, G. Giardina, S. Giovannella, F. Gonnella E. Graziani, F. Happacher, B. Höistad, L. Iafolla, E. Iarocci, M. Jacewicz, T. Johansson, A. Kupsc, J. Lee-Franzini, B. Leverington, F. Loddo, S. Loffedo, G. Mandaglio, M. Martemianov, M. Martini, M. Mascolo, R. Messi, S. Miscetti, G. Morello, D. Moricciani, P. Moskal, F. Nguyen, A. Passeri, V. Patera, I. Prado Longhi, A. Ranieri, C. F. Redmer, P. Santangelo, I. Sarra, M. Schioppa, B. Sciascia, M. Silarski, C. Taccini, L. Tortora, G. Venanzoni, R. Versaci, W. Wiślicki, M. Wolke, J. Zdebik

This is an Open Access article distributed under the terms of the Creative Commons Attribution License 2.0, which permits unrestricted use, distribution, and reproduction in any medium, provided the original work is properly cited. 


\section{$2 C \mathcal{P}$ violation in the neutral kaon system}

From the point of view of strong interactions the $K^{0}$ meson is a particle with a corresponding antiparticle $\vec{K}^{0}$. Violation of strangeness conservation by weak interaction allows for transitions like $K^{0} \rightarrow 2 \pi \rightarrow \bar{K}^{0}$ or $K^{0} \rightarrow 3 \pi \rightarrow \bar{K}^{0}$. Thus, the two strangeness eigenstates can oscillate one into another via the $\Delta S=2$, second order weak interactions. Neutral kaons decay mainly to the two and three - pion final states with a well defined $C \mathcal{P}$ eigenvalue, therefore propagation and decays of these particles is described in the basis of the $C \mathcal{P}$ operator eigenstates: $\left|K_{1}\right\rangle=\frac{1}{\sqrt{2}}\left(\left|K^{0}\right\rangle+\left|\bar{K}^{0}\right\rangle\right)$ with $\mathrm{CP}=+1$ and $\left|K_{2}\right\rangle=\frac{1}{\sqrt{2}}\left(\left|K^{0}\right\rangle-\left|\bar{K}^{0}\right\rangle\right)$ with $\mathrm{CP}=-1$. CP conservation would imply that $\left|K_{1}\right\rangle$ state is allowed to decay only to two pions with $C \mathcal{P}=1$, while the long living $\left|K_{2}\right\rangle$ decays only to three pions state with $C \mathcal{P}=-1$.

In 1964 the experiment by Christenson, Cronin, Fitch and Turlay, unexpectedly showed that the long - lived kaon can decay also to the two - pion final states with branching ratio of about 2 . $10^{-3}$ [1]. Thus, the neutral kaons states seen in nature are not the $C \mathcal{P}$ eigenstates defined before. However, they still can be expressed in the $\left(\left|K_{1}\right\rangle,\left|K_{2}\right\rangle\right)$ basis as: $\left|K_{L}\right\rangle=\frac{1}{\sqrt{1+|\epsilon|^{2}}}\left(\left|K_{2}\right\rangle+\epsilon\left|K_{1}\right\rangle\right)$, and $\left|K_{S}\right\rangle=\frac{1}{\sqrt{1+|\epsilon|^{2}}}\left(\left|K_{1}\right\rangle-\epsilon\left|K_{2}\right\rangle\right)$, where $\epsilon$ is the mixing parameter giving the weight of opposite $C \mathcal{P}$ eigenstate in the wavefunction. We can describe the $C \mathcal{P}$ symmetry breaking within the frame of two distinct mechanisms referred to as direct and indirect breaking. The indirect violation corresponds to the statement that the eigenstates of both the electroweak interactions are not exactly $C \mathcal{P}$ eigenstates but have small admixtures of the state with opposite $C \mathcal{P}$. It is also possible that $C \mathcal{P}$ violation occurs directly in the weak decays themselves.

Typically the $C \mathcal{P}$ violation in the neutral kaon sector is characterized in terms of the following amplitude ratios: $\eta_{+-}=A\left(K_{L} \rightarrow \pi^{+} \pi^{-}\right) / A\left(K_{S} \rightarrow \pi^{+} \pi^{-}\right) \cong \epsilon+\epsilon^{\prime}$, and $\eta_{00}=A\left(K_{L} \rightarrow \pi^{0} \pi^{0}\right) / A\left(K_{S} \rightarrow\right.$ $\left.\pi^{0} \pi^{0}\right) \cong \epsilon-2 \epsilon^{\prime}$, where the complex parameters $\epsilon^{\prime}$ and $\epsilon$ express the direct and indirect $C \mathcal{P}$ violation, respectively. In the framework of Standard Model an analogous CP mechanism affects the $K_{S}$ decays, for which the following amplitude ratios are defined: $\eta_{+-0}=A\left(K_{S} \rightarrow \pi^{+} \pi^{-} \pi^{0}\right) / A\left(K_{L} \rightarrow \pi^{+} \pi^{-} \pi^{0}\right) \cong$ $\epsilon+\epsilon_{+-0}^{\prime}$, and $\eta_{000}=A\left(K_{S} \rightarrow \pi^{0} \pi^{0} \pi^{0}\right) / A\left(K_{L} \rightarrow \pi^{0} \pi^{0} \pi^{0}\right) \cong \epsilon+\epsilon_{000}^{\prime}$. As in the case of two - pion decays, the ratio depends on the direct $C \mathcal{P}$ violation parameters related at the lowest order of the Chiral Perturbation Theory to the parameter of the two - pions decay: $\epsilon_{+-0}^{\prime}=\epsilon_{000}^{\prime}=-2 \epsilon^{\prime}$ [6]. While $\eta_{+-}$and $\eta_{00}$ have been measured with a good precision, the analogous parameters for $K_{S}$ are not well known [7]. In particular, the $K_{S} \rightarrow \pi^{0} \pi^{0} \pi^{0}$ decay had been never observed, and the branching ratio for this process is predicted to be very small in the Standard Model (about $2 \cdot 10^{-9}$ ). Therefore, studies of this decay demand high precision experiments like KLOE which will be briefly described in the next section.

\section{The KLOE experiment at DA $\Phi$ NE}

DAФNE is a $e^{+} e^{-}$collider optimized to work at a center of mass energy around the $\phi$ meson mass peak: $\sqrt{s}=1019.45 \mathrm{MeV}$ [8]. It consists of two rings in which 120 bunches of both, electrons and positrons, are stored. Particles are accelerated to final energy in the Linac (see left panel of Fig. 1), stored and cooled in the accumulator, and then transferred to the ring. The $e^{+}$and $e^{-}$beams collide with a small transverse momentum and produce $\phi$ mesons which are almost at rest $\left(\beta_{\phi} \approx 0.015\right)$. These decay mainly to $K^{+} K^{-}(49 \%), K_{S} K_{L}(34 \%), \rho \pi(15 \%)$ and $\eta \gamma(1.3 \%)$ [7]. The decay products are recorded using the KLOE detection setup, which is presented schematically in the right panel of Fig. 1. It consists of an about $3.3 \mathrm{~m}$ long cylindrical drift chamber with diameter of about $4 \mathrm{~m}$, which is surrounded by the electromagnetic calorimeter. The detectors are placed in the axial magnetic field of a superconducting solenoid of $0.52 \mathrm{~T}$.

The KLOE tracking chamber was designed to detect all charged secondary products from the $K_{L}$ decay and measure their properties with great precision. To minimize the $K_{L}$ regeneration, multiple Coulomb scattering and photon absorption the chamber is constructed out of carbon fiber composite with low $\mathrm{Z}$ and low density, and uses the gas mixture of helium (90\%) and isobutane (10\%). The KLOE drift 


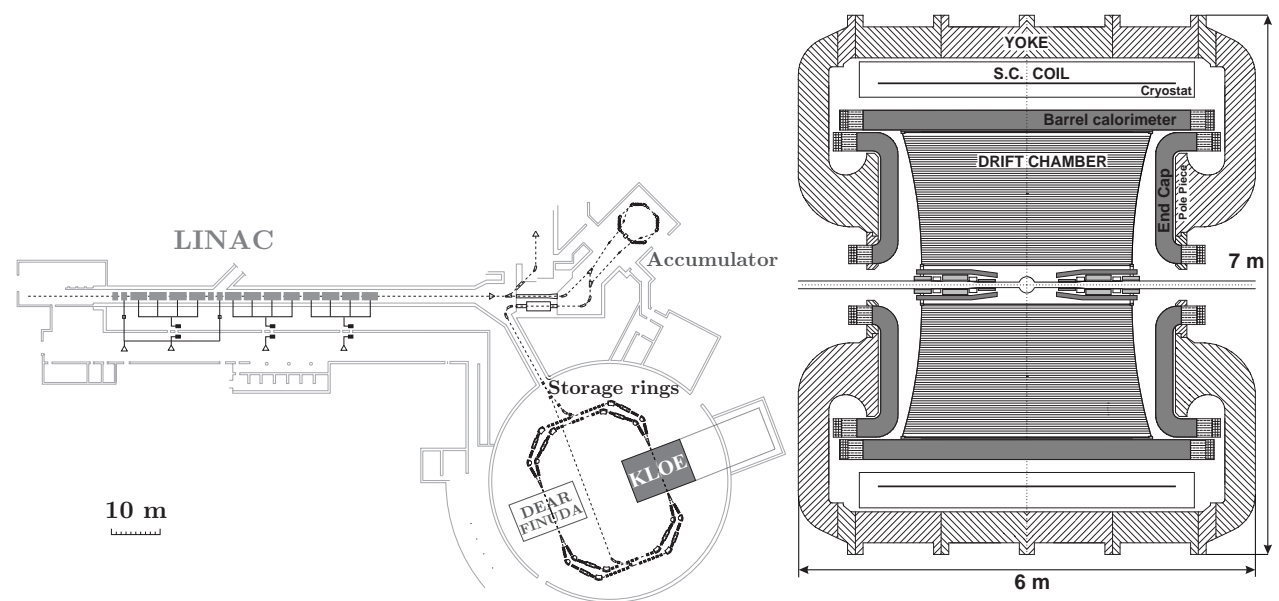

Fig. 1. Left panel: Scheme of the DAФNE complex; Right panel: Schematical view of the KLOE detector

chamber provides tracking in three dimensions with resolution in the bending plane of about $200 \mu \mathrm{m}$, resolution on the z-coordinate measurement of about $2 \mathrm{~mm}$ and of $1 \mathrm{~mm}$ on the decay vertex position. Momentum of the particle is determined from the curvature of its trajectory in the magnetic field with a fractional accuracy $\sigma_{p} / p=0.4 \%$ for polar angles larger than $45^{\circ}$ [8].

The KLOE electromagnetic calorimeter consists of a barrel built out of 24 trapezoidal shaped modules and two endcaps. Each of the modules is built out of $1 \mathrm{~mm}$ scintillating fibers grouped in cells of $4.4 \times 4.4 \mathrm{~cm}^{2}$ and embedded in $0.5 \mathrm{~mm}$ lead foils, and it is read out from both sides by set of photomultipliers. the showering processes [8]. This detector can measure particle energies and time with accuracies of $\sigma_{E} / E=5.7 \% / \sqrt{E[G e V]}$ and $\sigma(t)=57 \mathrm{ps} / \sqrt{E[\mathrm{GeV}]} \oplus 140 \mathrm{ps}$, respectively. Analysis of the signal amplitude distribution provides also the determination of the point where particles hit the calorimeter module with accuracy of about $1 \mathrm{~cm}$ in the plane transverse to the fiber direction. The longitudinal coordinate precision is energy dependent: $\sigma_{z}=1.2 \mathrm{~cm} / \sqrt{E[\mathrm{GeV}]}[8]$.

\section{Search for the $K_{S} \rightarrow \pi^{0} \pi^{0} \pi^{0}$ decay}

At KLOE kaons arising from the $\phi$ decay move at low speed with their relative angle close to $180^{\circ}$. Therefore, observation of a $K_{L}\left(K_{S}\right)$ decay ensures the presence of a $K_{S}\left(K_{L}\right)$ meson travelling in the opposite direction. The $K_{S}$ mesons are identified with high efficiency $(\sim 34 \%)$ via detection of the $K_{L}$ mesons which cross the drift chamber without decaying and then interact in the KLOE electromagnetic calorimeter (so called $K_{S}$ tag). The $K_{S}$ four - momentum vector is then determined using the measured position of the $K_{L}$ meson and the known momentum of the $\phi$ meson, which is estimated as an average of the momentum distribution measured using large angle $e^{+} e^{-}$scattering. The search for the $K_{S} \rightarrow 3 \pi^{0} \rightarrow 6 \gamma$ decay is then carried out by the selection of events with six photons whose momenta are reconstructed using time and energy measured by the electromagnetic calorimeter. Background for the searched decay originates mainly from the $K_{S} \rightarrow 2 \pi^{0}$ events with two spurious clusters from fragmentation of the electromagnetic showers (so called splitting) or accidental activity, or from false $K_{L}$ identification of $\phi \rightarrow K_{S} K_{L} \rightarrow \pi^{+} \pi^{-}, 3 \pi^{0}$ events. In the latter case charged pions from $K_{S}$ decays interact in the DA $\Phi$ NE low - beta insertion quadrupoles, ultimately simulating the $K_{L}$ interaction in the calorimeter, while $K_{L}$ decays close to the IP producing six photons [3]. To suppress this kind of background we first reject events with charged particles coming from the vicinity of the interaction region. Moreover, we cut also on the reconstructed velocity and energy of the tagging $K_{L}$ meson [9]. In the next stage of the analysis we perform a kinematic fit with 11 constraints: energy and momentum conservation, the kaon mass and the velocity of the six photons. Cutting on the $\chi^{2}$ of the fit considerably reduces the background from bad quality reconstructed events with a very good signal efficiency. In order to reject events with split and accidental clusters we look at the correlation between 
two $\chi^{2}$ - like discriminating variables $\chi_{2 \pi}^{2}$ and $\chi_{3 \pi}^{2} \cdot \chi_{2 \pi}^{2}$ is calculated by an algorithm selecting four out of six clusters, the best for the kinematic constraints of the two - body decay, it is therefore a test of the $K_{S} \rightarrow 2 \pi^{0} \rightarrow 4 \gamma$ hypothesis. The pairing of clusters is based on the requirement $m_{\gamma \gamma}=m_{\pi^{0}}$, and on the opening angle of the reconstructed pions trajectories in the $K_{S}$ center of mass frame. Moreover, we check the consistency of the energy and momentum conservation in the $\phi \rightarrow K_{S} K_{L}, K_{S} \rightarrow 2 \pi^{0}$ decay hypothesis [9]. The $\chi_{3 \pi}^{2}$ instead verifies the signal hypothesis by looking at the reconstructed masses of three pions. For every choice of cluster pairs we calculate the quadratic sum of the residuals between the nominal $\pi^{0}$ mass and the invariant masses of three photon pairs. In order to improve the quality of the photon selection using $\chi_{2 \pi}^{2}$, we cut on the variable $\Delta E=\left(m_{\Phi} / 2-\sum E_{\gamma_{i}}\right) / \sigma_{E}$ where $\gamma_{i}$ stands for the $\mathrm{i}-t h$ photon among those chosen in the $\chi_{2 \pi}^{2}$ estimator and $\sigma_{E}$ is the appropriate resolution. For $K_{S} \rightarrow 2 \pi^{0}$ decays plus two background clusters, we expect $\Delta E \sim 0$, while for $K_{S} \rightarrow 3 \pi^{0}$ $\Delta E \sim m_{\pi^{0}} / \sigma_{E}$. At the end of the analysis we cut also on the minimal distance between photon clusters to refine rejection of events with splitted clusters.

With preliminary cuts at the end of the analysis of $1.7 \mathrm{fb}^{-1}$ we count 0 candidates with 0 background events expected from Monte Carlo simulations of a sample two times larger than that of the data. A careful evaluation of systematics associated with background evaluation is being finalized.

From this preliminary analysis, we obtain the upper limit on the $K_{S} \rightarrow 3 \pi^{0}$ branching fraction of $B R\left(K_{S} \rightarrow 3 \pi^{0}\right)<2.7 \cdot 10^{-8}$ at $90 \%$ confidence level, almost five times lower than the latest published result [3].

\section{Summary and outlook}

As a result of the full KLOE data set analysis, gathered in the 2004 - 2005 data taking period, no events corresponding to the $K_{S} \rightarrow 3 \pi^{0}$ decay have been identified. Thus, we have set an upper limit for the $K_{S} \rightarrow 3 \pi^{0}$ branching ratio at $90 \%$ confidence level, which is almost five times lower than the latest published result [3]. However, the search for the $K_{S} \rightarrow 3 \pi^{0}$ decay will be continued by the KLOE -2 collaboration [10], which is extending the physics program of the predecessor. For the forthcoming run the KLOE performance have been improved by adding new subdetector systems: the tagger system for the $\gamma \gamma$ physics, the Inner Tracker based on the Cylindrical GEM technology and two calorimeters in the final focusing region [11]. These new calorimeters will increase the acceptance of the detector, while the new inner tracker will significantly reduce the contribution of the background processes with charged particles. Increasing the statistics and acceptance of the detector while significantly reducing the background gives the opportunity to observe the $K_{S} \rightarrow 3 \pi^{0}$ decay for the first time in the near future.

We acknowledge the support by the Polish National Science Center and by the Foundation for Polish Science.

\section{References}

1. J. H. Christenson, J. W. Cronin, V. L. Fitch, R. Turlay, Phys. Rev. Lett. 13, (1964) 138.

2. M. N. Achasov et al., Phys. Lett. B 459, (1999) 674.

3. F. Ambrosino et al., Phys. Lett. B 619, (2005) 61.

4. A. Angelopoulos et al., Phys. Lett. B 425, (1998) 391.

5. A. Lai et al., Phys. Lett. B 610, (2005) 165.

6. L. Maiani, N. Paver, in Maiani, L. (ed.) et al., The Second DAФNE physics handbook, (1995) 51.

7. K. Nakamura et al. (Particle Data Group), J. Phys. G 37, (2010) 075021.

8. F. Bossi et al., Riv. Nuovo Cim. 31, (2008) 531.

9. M. Silarski, PhD Thesis, Search for the CP symmetry violation in the decays of $K_{S}$ mesons using the KLOE detector, Cracow 2012.

10. G. Amelino-Camelia et al., Eur. Phys. J. C 68, (2010) 619.

11. D. Moricciani, PoS EPS -HEP2011 (2011) 198. 